Vietnam Journal of Mechanics, VAST, Vol. 27, No. 4 (2005), pp. 240-244

\title{
DEFORMATION OF MEDIUM HAVING STRING STRUCTURE
}

\author{
TRINH VAN KHOA \\ Hanoi Architectural University
}

\begin{abstract}
In this paper we constructed a model of plastic deformation of the medium having string structure. The knot theory was used to classify the plastic state.
\end{abstract}

\section{INTRODUCTION}

Knot theory was born in Scotland around the year of 1867. J.C. Maxwell, P.G. Tait and $\mathrm{W}$. Thomson were the founders of what that has become a knot theory. According to Thomson's theory of chemical elements all atoms are made of small knots formed by vortex lines of either which have to be kinetically stable. After that Alexander has performed the classification of the knot (knot invariant). Now once again knot problem is studding strongly thanks to the results of Jones. In the time one achieves important progress in the mathematics and the physics when one uses knot theory.

Concretely, in the paper [1] the connections between the theory of knot and statistical mechanics are shown and in the theory of knot there is the new device for considering precisely - soluble problem of statistical mechanics. In this case, in the evolutionary equation there is the dynamical symmetry. In simplest case, the determination of invariant of knot is equivalent to determination of function of distribution of probability in a critical point for two-dimension models. Ignoring the strict of mathematical requirement it is equivalent to definition of Feymann integral over a trajectory. That means, every Feymann diagram corresponds a knot invariant. It is the mean idea of Witten. Since, in the Chern-Simons-Witten theory the statistical sum is covariant - equivalent to knot invariant ("Link invariant"). A matter of course, the Wilson line was identified with a strings. In research of two-dimension model a condition of integrability is connected to a subgroup of braid group (in the Yang-Baxter equation). What do we receive when we use this idea. In paper [7] we used statistical sum of a Chern-Simons-Witten type for constructing a model of plastic deformation. At this case the Ilyushin trajectory of deformation was identified with a knot. However, our problem is possible to simplify, when we are having admitted the existence of string structure, in which a deformation trajectory of a string also is a string. Polymer medium is an medium of such type. For simplicity we shall consider only two-dimension problem. For polymer medium A.Iu. Grosberg considered the statistical sum with the help of the concept of the entropy of a knot (possible phase volume) and a maximum swollen representation of the knot [8]. S. K. Netraev, in paper [9], used algebraic Kauffman invariant of the knot for demonstration of direct connection of topological character with thermodynamic properties of two-dimension Post model in frozen, or in the dynamical disorder with a constant interaction [9].

In this paper we use the representation method of braid groups to design Feymann integral for the distribution of probability in the problem of plastic deformation in the synergetic formalism. It will allow to see the connection of distribution of probabilities in our paper and the Gauss distribution in papers [5, 10]. 


\section{DEFORMATION AS THE PROCESS OF THE SPONTANEOUS FORMATION OF THE STRUCTURE}

Last years, one has saved huge results of the experiments, allowing to formulate the new approaches to problems of plasticity. There is a special attention, that one deserves the synergetic approach, considering a deformable firm body as an open, strongly nonequilibrium system, and a plastic deformation - as a dissipative process lowering the level of elastic stress of medium $[2,3]$. On the essence, it is process of birth and distribution of soliton with certain topological characteristics [7]. Now the wave character of plastic deformation of firm bodies does not cause doubts. According to the synergetic approach the solution of the Fokker - Planck equation is a density of probability distribution of transition to plasticity. However, using potential character of a process of deformation, instead of the Fokker - Planck equation it is possible to use the Schrodinger equation [7]. So, we shall consider the equation of deformation process as the Schrodinger equation:

$$
\frac{\partial f}{\partial t}=\frac{\partial^{2}}{\partial e^{2}} f-\frac{W(e)}{T} f
$$

where $W(e)$ is a potential of deformation as free energy, $T$-temperature. It is possible to write down the solution as Feymann integral [8]:

$$
\begin{aligned}
& f\left(e\left(t_{0}\right), t_{0} ; e(t), t\right)=\mathcal{N} \int \exp \left\{-i \int_{t_{0}}^{t} d \tau G\right\} d e(t), \\
& G=\left[\frac{W(e(\tau))}{T}+\frac{\dot{e}^{2}(\tau)}{2}\right],
\end{aligned}
$$

where the point above $e$ designates derivative in time and $N$ is an integral constant.

\section{THE PROPAGATION OF PLASTIC DEFORMATION}

In the mechanics of continuous medium we investigate the string in the role of the trajectory of deformation. For simplicity let's consider only 2-dimension case, when trajectories are located on a surface in the Euclide space. Thus it is possible to describe configuration space of system of $n$ strings as

$$
M_{n}=\left\{\left(Z_{1}, \ldots, Z_{n}\right): Z_{i} \neq Z_{j}, i \neq j\right\} .
$$

The topology of such configuration space is non-trivial. The homotopy class is determined through the "winding number" $n$. Let's consider two strings $a$ and $b$. The number $n$ is determined by Gausse integral for any part of a curve $C$, which does not pass through string $b$, located in a point $\xi[5]$ :

$$
n=\frac{1}{2 \pi i} \int_{C} \frac{d z}{z-\xi} .
$$

However, generally in two-dimension coordinates, instead of the "winding number" $n$ it is possible to determine a "winding angle" $\theta$ of the curve $a$ (curve $C$ ) for the string $b$. It is the difference of the initial and final angle plus angles of $n$ winding circle $(2 \pi n)$ :

$$
\theta=\theta_{1}-\theta_{o}+2 \pi n
$$


where $\theta_{0}$ and $\theta_{1}$ are corners corresponding to initial and final points of a curve. Every string has the itself internal space. We can choose the internal space as Lee group $G$. So, it is possible to generalize "number of revolutions" (winding number), which will receive the following value in this internal space. If we denote $T_{a}, T_{b}$ being presentations of elements $a, b$, then winding character of these elements is in the form of $n T_{a} \otimes T_{b}$, where $n$ - winding number and $T_{a} \otimes T_{b}$ is tensor product of the presentations $T_{a}, T_{b}, \operatorname{sign}(C)$ denote the direct of the curve. Then we receive the following value $[4,12]$ :

$$
\begin{aligned}
& \theta=\operatorname{sign}(C)\left|\theta_{1}-\theta_{o}\right|+2 \pi w, \\
& w=n T_{a} \otimes T_{b}=\frac{1}{2 \pi i} \int_{C} \frac{d z}{z-\xi} T_{a} \otimes T_{b} .
\end{aligned}
$$

We carry out the parametrization of a trajectory through $z(t), t_{0} \leq t \leq t_{1}$, herein it is possible to consider the Feymann propagation of the string $a$ from place $z_{0}=z\left(t_{0}\right)$ to a place $z_{1}=z\left(t_{1}\right)$. Then similarly to paper $[4,12]$, it is possible to write the Feymann propagator for the homotopy class $l$ as

$$
K_{l}\left(z_{1}, t_{1}, z_{0}, t_{0}\right)=\int D_{l} z(t) D_{l} \bar{z}(t) \exp \left(i \int_{t_{0}}^{t_{1}} G d t\right) \delta\left(2 \pi l T_{a} \otimes T_{b}-\theta\right)
$$

where $G$ was given in (3.2). In the integral presentation Dirac function $\delta$ in (3.5) for looks like

$$
\begin{aligned}
\delta\left(2 \pi l T_{a} \otimes T_{b}-\theta\right)= & \int \frac{d k d \bar{k}}{4 \pi^{2}} \exp [-i(k \phi+\bar{k} \phi)] \times \\
& \times P \exp \left\{i\left[2 \pi k\left(l T_{a} \otimes T_{b}-w\right)\right]+c . c\right\}
\end{aligned}
$$

where c.c- is an approximation of the high order of the Fourier's expansion. This is the description in the form of functional integral of topological property of configuration spaces. Because there was a self-organizing in the process of plastic deformation, then non-trivial topological "interaction" has appeared between strings. Substituting (3.6) in (3.5) we shall receive

$$
\begin{aligned}
K_{l}\left(z_{1}, t_{1}, z_{0}, t_{0}\right)= & \int \frac{d k d \bar{k}}{4 \pi^{2}} \exp [-i(k \phi+\bar{k} \phi)] \times \overline{K_{l}}\left(z_{1}, t_{1}, z_{0}, t_{0} ; k, \bar{k}\right) \\
\overline{K_{l}}\left(z_{1}, t_{1}, z_{0}, t_{0} ; k, \bar{k}\right)= & \int D_{l} z(t) D_{l} \bar{z}(t) \exp \left(i \int_{t_{0}}^{t_{1}} G d t\right) \times \\
& \times \exp \left\{i \int_{t_{0}}^{t_{1}}\left[k\left(\frac{i z}{z-\xi}+2 \pi l\right) T_{a} \otimes T_{b}+c . c\right] d t\right\} .
\end{aligned}
$$

Similarly, we can generalize (3.7) for the homotopical class $\left(l_{1}, \ldots, l_{i-1}, l_{i+1}, \ldots, l_{n}\right)$, we 
receive Feymann propagator for a trajectory $i$, having representation $T_{i}$ :

$$
\begin{aligned}
K_{l i}\left(z_{1 i}, t_{1}, z_{0 i}, t_{0}\right)= & \int \frac{d k d \bar{k}}{4 \pi^{2}} \exp \left[-i \sum_{i, j=1}^{n}\left(k \phi_{i j}+\bar{k} \phi_{i j}\right)\right] \times \\
& \times \bar{K}_{l i}\left(z_{l i 1}, t_{1}, z_{l i 0}, t_{0} ; k, \bar{k}\right) \\
\bar{K}_{l i}\left(z_{i 1}, t_{1}, z_{i 0}, t_{0} ; k, \bar{k}\right)= & \int D_{l} z(t) D_{l} \bar{z}(t) \exp \left(i \int_{t_{0}}^{t_{1}} G d t\right) \times \\
& \times \exp \left\{i \int_{t_{0}}^{t_{1}}\left[k \sum_{j=1, j \neq i}^{n}\left(\frac{i z_{i}}{z_{i}-z_{j}}+2 \pi l_{j}\right) T_{i} \otimes T_{j}+\text { c.c }\right] d t\right\} .
\end{aligned}
$$

where we denote a difference of initial and final corners of a trajectory $i$ concerning a trajectory $j$ through $\phi_{i j}$ :

$$
\phi_{i j}=\operatorname{sign}\left(C_{i}\right)\left|\theta_{i j 1}-\theta_{i j 0}\right| \text {. }
$$

Thus; we have receiving the density of probability distribution of transfer to plasticity in the form:

$$
f=\int K_{i j} d e
$$

\section{DISCUSSION}

The medium with string structure is an medium such as polymer or the material of composites [5]. In an usual situation this medium is considered as Neutral. The research of a condition of deformation of such medium was executed on Flory model in [5]. In paper [6] the influence of topology on the deformation was considered. We identify a string with an ideal polymer circuit. In any point of a circuit from definition of deformation we can receive

$$
<d l^{2}>-<d l^{2}>=<2 e \gg<d l^{2}>
$$

where $\ll \gg$ - average meaning(importance) on distribution of probability of deformation, $<>$ - average meaning(importance) on Gauss distribution for ideal polymer. It means, that it is possible to receive Gauss Distribution through the distribution of probability of deformation and on the contrary. It is necessary to notice, that for each group $G$ and its representation there is a statistical sum or a function of distribution. The topological character of this group plays a main role in the process of taking shape plastic structure. In the classical theory of plasticity each trajectory determines the appropriate plastic condition. The interaction between the trajectories did not take into account. As a matter of fact, in the situation nontrivial topology exists because of the interactions of strings during self-organizing. So, the string theory of plasticity is constructed under the following schema. Let in beginning in body there are elastic strings or long elastic areas of a type of strings (for example, the macromolecular of polymers [5]). In a condition of self-organizing each string has group and its.representation. For each type of interaction of strings one statistical sum or Feymann integral is determined, ie. there is the classification of a plastic state with the help of knot invariant. The probability of elasticity - plasticity transition is determined due to this propagator. 


\section{REFERENCES}

1. V. F. Jones, A polynomial invariant for links via von Newmann algebras, Bull. Am. Math. Sos. 12 (1985) 103.

2. V. E. Panin, Volnovaia priroda plastitreskoi deformasi, Izv. vuzov, Physics (1) (1987) c.3.

3. V. C. Ivanova, Mechanika i synergetika ustalostnogo razrusenia, FChMM 21 (1986) 120.

4. C. H. Lai, C. Ting, Path integral representation of the Artin braid group, Phys. Lett. B 265 (1991) 341.

5. A. Yu. Grosberg, A. R. Khokhlov, Statistical Physics of Macromolecular, Science M: 1989.

6. F. F. Ternovski, A. R. Khokhlov, Molekuliarnaia teopia vucokoelastitrnosti polimernuch setok c utretom topologitreckoi ogranitrenia, JETF 90 (4) (1986) 1249.

7. V. K. Trinh, Doklad of RAN, The modern analysis and its application, Procceding of VZMS-2000 Conference 378 (3) (2001).

8. A. Yu. Grosberg, Disordered polymers, Ucpexi of Physics 167 (2) (1997).

9. C. K. Nechaev, Problems of probabilistics topology: statistics of knot and noncommutative random walks, Ucpexi of Physics 167 (4) (1997).

10. M. D. Frank-Kameneski, A. V. Vologodski, Mechanics of polymers, Ucpexi of Physics 134 (1981) 641.

11. A. L. Kholodenko, T. A. Vilgis, Some geometrical and topological problem in polymer physics, Phys. Reports 298 (1998) $251-370$.

12. F. Pham, Homotopy Theory and Feymann Integral, 1974.

Received November 30, 2004

Revised October 28, 2005

\section{BIẾN DẠNG CƯA MÔI TRƯờNG CÓ CẤU TRƯC DÂY}

Trong bài báo này chúng tôi xây mô hình biến dạng dẻo của môi trường có cấu trúc dây. Lý thuyết dây đã được sử dựng để phân loại các trạng thái. 